\title{
Enhanced signal of astrophysical tau neutrinos propagating through Earth
}

\author{
John F. Beacom* \\ NASA/Fermilab Astrophysics Center, Fermi National Accelerator Laboratory, Batavia, Illinois 60510-0500 \\ Patrick Crotty ${ }^{\dagger}$ \\ Department of Physics, University of Chicago, Chicago, Illinois 60637 \\ Edward W. Kolb \\ NASA/Fermilab Astrophysics Center, Fermi National Accelerator Laboratory, Batavia, Illinois 60510-0500, \\ Department of Astronomy and Astrophysics, University of Chicago, Chicago, Illinois 60637, \\ and TH Division, CERN, CH-1211 Geneva 23, Switzerland \\ (Received 26 November 2001; published 9 July 2002)
}

\begin{abstract}
Earth absorbs $\nu_{e}$ and $\nu_{\mu}$ of energies above about $100 \mathrm{TeV}$. As is well known, although $\nu_{\tau}$ will also disappear through charged-current interactions, the $\nu_{\tau}$ flux will be regenerated by prompt tau decays. We show that this process also produces relatively large fluxes of secondary $\bar{\nu}_{e}$ and $\bar{\nu}_{\mu}$, greatly enhancing the detectability of the initial $\nu_{\tau}$. This is particularly important because at these energies $\nu_{\tau}$ is a significant fraction of the expected astrophysical neutrino flux, and only a tiny portion of the atmospheric neutrino flux.
\end{abstract}

DOI: 10.1103/PhysRevD.66.021302

PACS number(s): 95.85.Ry, 14.60.Pq, 96.40.Tv

\section{INTRODUCTION}

A variety of astrophysical and exotic sources are expected to produce large fluxes of ultrahigh-energy particles, including photons, protons, and neutrinos. Sources such as active galactic nuclei and gamma-ray bursts are at high redshifts, and the photons and protons will be attenuated by scattering on cosmic radiation backgrounds. The protons may also be deflected by magnetic fields. However, neutrinos from distant sources will neither be attenuated nor deflected, thus allowing true neutrino astronomy of the high-redshift universe. Further, it is now known that about one-third of the energy density of the universe is accounted for by particles outside the standard model. Among the possibilities are variants of superheavy dark matter, which may produce ultrahigh-energy particles from their annihilations or decays. Such signals would be most readily observed from local sources. The existence of cosmic rays with energies up to and above $10^{20} \mathrm{eV}$ is a powerful argument for the existence of ultrahigh-energy astrophysical neutrinos. With the upcoming $\mathrm{km}$-scale detectors, a new era of neutrino astronomy will begin, and the energies, directions, and flavors of astrophysical neutrinos will provide important clues to the most violent astrophysical objects and the nature of particle dark matter $[1,2]$.

While these detectors can suppress downgoing atmospheric muons, atmospheric neutrinos are a much more challenging background. Like astrophysical neutrinos, these can pass through Earth and create upgoing muons (for example). They are produced in the decays of secondaries produced by the collisions of cosmic-ray protons with Earth's atmosphere.

\footnotetext{
*Email address: beacom@fnal.gov

†Email address: prcrotty@oddjob.uchicago.edu

‡Email address: rocky@fnal.gov
}

The decays $\pi^{+} / K^{+} \rightarrow \mu^{+} \nu_{\mu}$ and $\mu^{+} \rightarrow e^{+} \nu_{e} \bar{\nu}_{\mu}$ and their charge conjugates produce fluxes in the flavor ratio $\nu_{e}: \nu_{\mu}: \nu_{\tau} \simeq 1: 2: 0$, where neutrinos and antineutrinos have been combined. These ratios vary with energy and angle when the production of other mesons and their decay lengths relative to the slant depth of the atmosphere are taken into account. In particular, at the highest neutrino energies, there is a tiny relative $\nu_{\tau}$ flux from charm decay [3]. The atmospheric neutrino flux falls roughly as $E^{-3}$, whereas general considerations for astrophysical neutrino fluxes predict shallower slopes, and hence that they will dominate above some energy. For diffuse fluxes, this crossover is thought to be at least $10 \mathrm{TeV}$ (point and/or transient sources can be identified at lower energies) [1,2].

Neutrino oscillations have an important consequence for suppressing the atmospheric neutrino background. The Super-Kamiokande atmospheric neutrino results [4] strongly favor $\nu_{\mu} \leftrightarrow \nu_{\tau}$ oscillations with $\sin ^{2} 2 \theta \simeq 1$ and $\Delta m^{2} \simeq 3$ $\times 10^{-3} \mathrm{eV}^{2}$. At the high energies considered here, $\nu_{\mu} \leftrightarrow \nu_{\tau}$ oscillations will never occur for atmospheric neutrinos, and their flavor ratios will reflect the production mechanism only. In the astrophysical production scenarios, the flavor ratios are also thought to be 1:2:0 (again combining neutrinos and antineutrinos), reflecting their production by ultrahighenergy protons. But for the astrophysical sources, $\nu_{\mu} \leftrightarrow \nu_{\tau}$ mixing will always be complete because of the long path lengths, and so these ratios become 1:1:1. In some exotic models $[5,6]$, the initial $\nu_{\tau}$ flux is large; in any case, a large $\nu_{\tau}$ component in the astrophysical neutrino flux is guaranteed.

This simple fact is very important for the detection of astrophysical neutrinos above the atmospheric neutrino background. This is because $\nu_{e}$ and $\nu_{\mu}$ above about $100 \mathrm{TeV}$ will be absorbed by Earth, whereas the absorbed $\nu_{\tau}$ flux will be regenerated by prompt tau decays [7]. We point out a new effect: that the regeneration of the $\nu_{\tau}$ flux also creates rela- 


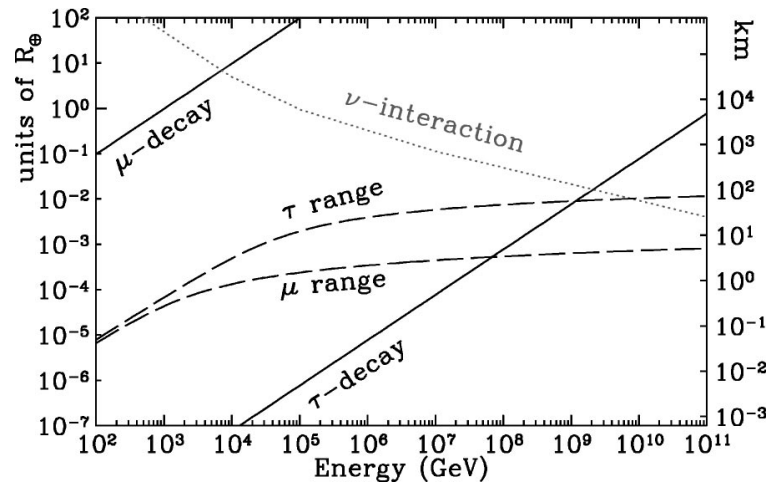

FIG. 1. Some important length scales, in units of Earth radii and $\mathrm{km}$, versus the energy in $\mathrm{GeV}$ for the neutrino or charged lepton. Solid lines: the $\mu$ and $\tau$ decay lengths, i.e., the distances over which a fraction $e^{-1}$ of the initial fluxes would survive (ignoring energy loss). Dashed lines: the $\mu$ and $\tau$ ranges, i.e., the distances in standard rock $\left(22 \mathrm{~g} \mathrm{~mol}^{-1}\right)$ at a density $8 \mathrm{~g} \mathrm{~cm}^{-3}$ over which they would be fully stopped by their electromagnetic interactions (ignoring decays). Dotted line: the neutrino interaction length (the same for all flavors), i.e., the distance at a density $8 \mathrm{~g} \mathrm{~cm}^{-3}$ over which a fraction $e^{-1}$ of the initial flux would survive. With the exception of the Glashow resonance for $\bar{\nu}_{e}$ at $6.3 \mathrm{PeV}$, this figure is the same for antiparticles. While the density varies significantly with the nadir angle, $8 \mathrm{~g} \mathrm{~cm}^{-3}$ is representative.

tively large secondary fluxes of $\bar{\nu}_{e}$ and $\bar{\nu}_{\mu}$. These make the detection of astrophysical sources significantly easier, and must be accounted for to properly deduce the source spectra and flavor composition.

\section{NEUTRINO PROPAGATION IN EARTH}

Above about $100 \mathrm{TeV}$, all neutrino flavors have a high probability of interacting in Earth via neutrino-nucleon scattering, with a total cross section $\sigma \propto E^{0.5}$ [8]. Except for $\bar{\nu}_{e}$ $+e^{-} \rightarrow W^{-}$at the Glashow resonance $(6.3 \mathrm{PeV})$, neutrinoelectron scattering is irrelevant. Charged-current (CC) and neutral-current (NC) interactions on nucleons occur in the ratio $0.71: 0.29$, and in both cases, the surviving lepton carries about $75 \%$ of the initial neutrino energy [8]. The cross sections for neutrinos and antineutrinos are approximately equal. The neutrino interaction length is shown in Fig. 1; it is the same for all flavors since even tau mass threshold effects are negligible.

In the more likely $\mathrm{CC}$ interaction, the critical question is the subsequent fate of the charged lepton. An initial $\nu_{e}$ will be removed from the beam and the resulting electron very quickly brought to rest. With an initial $\nu_{\mu}$, the produced muon will eventually decay. However, the laboratory-frame decay length for the muon is always much longer than its range, the distance over which its electromagnetic energy losses bring it to rest. Thus the neutrinos from its decay will be below $50 \mathrm{MeV}$, and of no further interest here. The decay length and range for muons are shown in Fig. 1.

As first noted by Ritz and Seckel [9], the situation is very different for $\nu_{\tau}$ since, at all but the highest energies, the tau decay length is less than its range, so that the tau will decay in flight without significant energy loss; see Fig. 1. Tau decays occur by many branches, but all contain a $\nu_{\tau}$. This regenerated $\nu_{\tau}$ carries a fraction about $0.75 \times 0.4 \simeq 1 / 3$ of the initial $\nu_{\tau}$ energy, where 0.4 is for $\nu_{\tau}$ from tau decay $[10,11]$. Ritz and Seckel considered weakly interacting massive particle (WIMP) annihilation in the Sun, and the propagation of the produced neutrinos. The utility of this regeneration effect for the detection of astrophysical neutrinos was not noted until a decade later by Halzen and Saltzberg [7].

The regeneration process will continue (along with the less frequent $\mathrm{NC}$ scatterings) until the $\nu_{\tau}$ energy has been moderated to the energy such that the neutrino interaction length is comparable to the remaining distance in Earth. At nadir angle $\psi=0^{\circ}$, such that the neutrino will cross the full diameter of Earth, the transparency energy is about $40 \mathrm{TeV}$ (at larger nadir angles, neutrinos at higher energies can pass through without scattering). The distribution of $\nu_{\tau}$ energies around the transparency energy is characteristically lognormal, with a one-sigma width of approximately one decade. Thus all of the initial $\nu_{\tau}$ will emerge at relatively high energies.

Future km-scale astrophysical neutrino detectors are designed for the detection of $\nu_{e}$ (via an electromagnetic shower) or $\nu_{\mu}$ (via the track of a penetrating muon). The detection of $\nu_{\tau}$ is much more difficult. Learned and Pakvasa [12] have shown that $\nu_{\tau}$ may be detected by a "doublebang" signature if the tau lepton production and decay, each accompanied by a large shower, are well separated but both occur in the detector. The double-bang events will be observable only in a narrow range around several $\mathrm{PeV}$, and only at large nadir angles, so other techniques for $\nu_{\tau}$ detection are needed.

This is precisely the point of the signal proposed by Halzen and Saltzberg [7]. In their scheme, the $\nu_{\tau}$ interacts outside the detector and the tau created decays as $\tau^{-}$ $\rightarrow \mu^{-} \bar{\nu}_{\mu} \nu_{\tau}$, where the muon, which has a long range, is seen in the detector. These $\nu_{\tau}$ events can be separated from $\nu_{\mu} \mathrm{CC}$ events (which also produce a muon) on a statistical basis, using the fact that the $\nu_{\tau}$ nadir angle distribution will be unchanged by passage through Earth, whereas that for $\nu_{\mu}$ will show exponential absorption (as will $\nu_{e}$ ). Additionally, if the incoming neutrino spectrum is shallower than about $E^{-2}$, the pileup near the transparency energy may be visible $[7,10]$. However, it should be noted that the above tau branching ratio of $18 \%$ reduces the detectability of the $\nu_{\tau}$ flux compared to a $\nu_{\mu}$ flux at the same energy, both taken just below the detector. While the muon in the $\nu_{\tau}$-induced case will have an energy roughly 0.4 of that in the $\nu_{\mu}$-induced case because of the tau decay $[10,11]$, the muon range and hence detectability varies only slowly with energy (see Fig. 1).

\section{SECONDARY ANTINEUTRINOS}

While expected to be a significant component of the astrophysical neutrino flux, and the only component which is not attenuated in Earth, $\nu_{\tau}$ are not easily detected. However, we point out a new effect that significantly improves their detectability. In each step of the regeneration process $\nu_{\tau}$ 
$\rightarrow \tau \longrightarrow \nu_{\tau}$ described above, the tau lepton decay will always produce a $\nu_{\tau}$. Additionally, $18 \%$ of decays are $\tau \rightarrow \nu_{\tau} \mu \bar{\nu}_{\mu}$ and $18 \%$ are $\tau \rightarrow \nu_{\tau} e \bar{\nu}_{e}$. In the laboratory frame, on average $\nu_{\tau}$ and $\mu$ each carry a fraction 0.4 of the tau energy and $\bar{\nu}_{\mu}$ carries a fraction $0.2[10,11]$. Secondary $\nu_{\mu}$ and $\nu_{e}$ are also produced by initial $\bar{\nu}_{\tau}$ (expected to have a flux comparable to the initial $\left.\nu_{\tau}\right)$. These secondary antineutrinos $\left(\bar{\nu}_{e}, \bar{\nu}_{\mu}\right)$ and neutrinos $\left(\nu_{e}, \nu_{\mu}\right)$ have not been taken into account before, but they have a surprisingly large effect on the detectability of $\nu_{\tau}$ and $\bar{\nu}_{\tau}$.

The number of regeneration steps for $\nu_{\tau}$ is approximately $N=\log \left(E_{i} / E_{T}\right) / \log 3$, where $E_{i}$ is the initial energy and $E_{T}$ is the transparency energy. With more steps, there are more opportunities to create secondary $\bar{\nu}_{\mu}$ and $\bar{\nu}_{e}$. However, those created farther from the detector have a greater chance of being absorbed. On each step the secondary antineutrinos will carry about $1 / 6$ of the initial $\nu_{\tau}$ energy, reflecting the energy lost in the CC scattering and the tau decay.

It is generally assumed that all scatterings and decays are collinear. However, as the number of regeneration steps increases, the cumulative non-collinearity could be large enough to blur astrophysical point sources. If the initial neutrino has energy $E_{\nu}$, simple considerations indicate that for $\nu_{\tau} \rightarrow \tau$, the scattering angle is about $1 \% \sqrt{E_{\nu} / \mathrm{TeV}}$, and for $\tau \rightarrow \nu_{\tau}$, the decay angle is about $0.2^{\circ} /\left(E_{\nu} / \mathrm{TeV}\right)$. The decay angle can thus be neglected compared to the scattering angle. In order for the number of scatterings to be nonzero, $E_{\nu}$ must be above about $100 \mathrm{TeV}$, so that on the last step the angular deviation is about $0.1^{\circ}$. Taking previous regeneration steps into account gives a maximum deviation of about $0.3^{\circ}$. This is below the expected $1^{\circ}$ reconstruction resolution of the proposed detectors [2], and so astrophysical point sources should not be blurred by $\nu_{\tau}$ regeneration.

We can estimate the secondary $\bar{\nu}_{e}$ and $\bar{\nu}_{\mu}$ number flux produced by a $\nu_{\tau}$ beam by making the following crude assumptions: (i) $\nu_{\tau}$ do not lose energy in their CC or NC interactions; (ii) $\bar{\nu}_{e}$ or $\bar{\nu}_{\mu}$ which interact are removed from the beam; and (iii) $\bar{\nu}_{e}$ or $\bar{\nu}_{\mu}$ from tau decay are produced with the same energy as the initial $\nu_{\tau}$. Consider a beam of astrophysical neutrinos in the expected ratio $\nu_{e}: \nu_{\mu}: \nu_{\tau}=1: 1: 1$, each with a flux $F_{0}$, and all at an energy $E_{0}$. Define $f$ as the fraction of $\nu_{\tau}$ interactions that produce a $\bar{\nu}_{e}$ or $\bar{\nu}_{\mu}: f=0.71$ $\times 0.18=0.13$, where the first factor is the CC/NC fraction, and the second factor is the relevant tau-decay branching ratio. The secondary $\bar{\nu}_{\mu}$ flux from tau decay obeys the equation

$$
\frac{d}{d x} F_{\nu_{\mu}}(x)=\frac{f}{\lambda} F_{0}-\frac{1}{\lambda} F_{\nu_{\mu}}(x),
$$

where $x$ is the distance through Earth at the chosen nadir angle. The first term represents the creation of $\bar{\nu}_{\mu}$ from the $\nu_{\tau}$ flux, and the second represents their subsequent absorption. Note that the interaction length $\lambda$ is the same for both flavors. Thus, in this simple estimate, the fluxes after propagation through Earth are

$$
\begin{aligned}
& F_{\nu_{e}} / F_{0}=F_{\nu_{\mu}} / F_{0}=e^{-x / \lambda} ; F_{\nu_{\tau}} / F_{0}=1 \\
& F_{\nu_{e}} / F_{0}=F_{\nu_{\mu}} / F_{0}=f\left(1-e^{-x / \lambda}\right) ; F_{\nu_{\tau}} / F_{0}=0 .
\end{aligned}
$$

The initial $\nu_{e}$ and $\nu_{\mu}$ fluxes are exponentially depleted. However, the flux associated with the initial $\nu_{\tau}$ is increased due to the secondary $\bar{\nu}_{e}$ and $\bar{\nu}_{\mu}$. For large $x$, corresponding to more $\nu_{\tau}$ regeneration steps, the increased production of $\bar{\nu}_{e}$ and $\bar{\nu}_{\mu}$ is balanced by their increased absorption. For the expected initial antineutrinos (with the same fluxes and flavor ratios as for neutrinos), similar results are obtained for the production of secondary neutrinos, and they must be added to the above. For small $x$, the initial $\nu_{e}, \nu_{\mu}, \bar{\nu}_{e}, \bar{\nu}_{\mu}$ fluxes will survive, whereas for large $x$, they will be absorbed but replaced by the approximately constant secondary fluxes created by $\nu_{\tau}$ and $\bar{\nu}_{\tau}$. Thus even for large energies and/or small nadir angles, the fluxes of $\nu_{e}, \nu_{\mu}, \bar{\nu}_{e}, \bar{\nu}_{\mu}$ never vanish, contrary to previous predictions.

We have calculated the secondary $\bar{\nu}_{e}$ and $\bar{\nu}_{\mu}$ production with a Monte Carlo code that simulates the passage of highenergy neutrinos through Earth. We assume collinear propagation for all particles followed, as justified above. The code starts with an initial neutrino and randomly samples its interaction probability at each step in distance, using the CC and NC total cross sections from Gandhi, Quigg, Reno, and Sarcevic [8]. In each interaction, we sample the outgoing charged lepton or neutrino energy around the average inelasticity values $\langle y\rangle$ given in Ref. [8]. At these energies, $\langle y\rangle$ is the same for $\mathrm{CC}$ or $\mathrm{NC}$ interactions, and the same for neutrinos or antineutrinos. Accordingly, for the distributions about $\langle y\rangle$ we use the $d \sigma / d y$ distribution given in Ref. [8] for neutrino-nucleon $\mathrm{CC}$ scattering.

After a NC interaction, the neutrino is followed until the next interaction. After a CC interaction, if a muon or tau is created, it is followed. For their energy loss, we use the results of Ref. [13], and for their decays we use the laboratory-frame decay probability distributions. Any electrons created are assumed stopped. For the laboratory-frame $\nu_{\tau}$ distribution from tau decays, summed over all branches and including the tau polarization, we use the distributions given in Refs. [10,11]. In tau decays leading to $\bar{\nu}_{e}$ or $\bar{\nu}_{\mu}$, we use the distributions for those branches [10,11]. There are many tau decay branches that yield charged pions or kaons. However, these mesons will be stopped before they decay, so that the produced neutrinos are at low energies and hence ignored. For all particles, we impose a low-energy cutoff at $100 \mathrm{GeV}$, though the results are insensitive to the cutoff as long as it is well below the transparency energy.

In Fig. 2 (cf. Fig. 2 of Ref. [7]), we plot the numerical results for the emergent $\nu_{\mu}$ and $\nu_{\tau}+\bar{\nu}_{\mu}$ fluxes using several initial energies. A very small fraction (about $1 \%$ ) of $\nu_{\tau}$ either downscatter to very low energies by repeated NC interactions or the tau lepton emerges from Earth; these are neglected in Fig. 2. The results are in general agreement with the analytic estimates, though the $\bar{\nu}_{\mu}$ flux is about twice as large as the analytic estimate. The principal reason for this is 


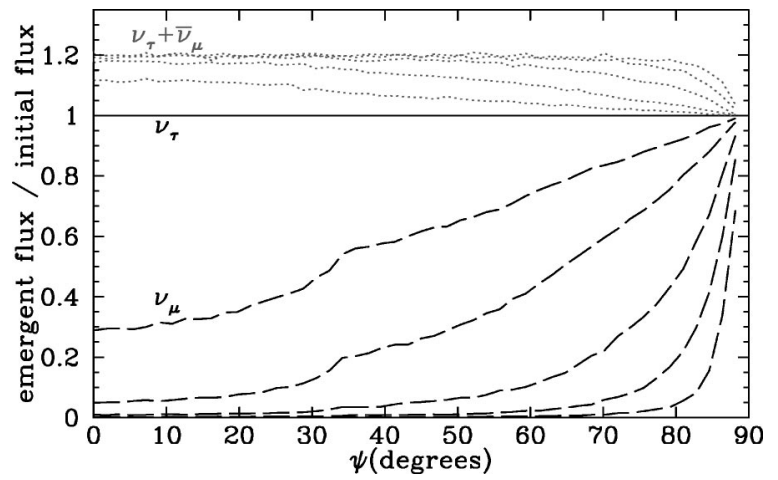

FIG. 2. The surviving fluxes associated with the initial $\nu_{\tau}$ and $\nu_{\mu}$ fluxes versus the nadir angle for a variety of energies. The results were calculated with our Monte Carlo code, and the slight jaggedness in the curves is due to statistical fluctuations. The initial $\nu_{\mu}$ flux is depleted ( $\nu_{e}$ would be the same), and the flux associated with the initial $\nu_{\tau}$ is increased due to the secondary $\bar{\nu}_{\mu}$ (and the equal $\bar{\nu}_{e}$ component, not shown). The initial energies are $10^{5}, 10^{6}$, $10^{7}, 10^{8}$, and $10^{9} \mathrm{GeV}$, going from top to bottom for the $\nu_{\mu}$ fluxes and bottom to top for the $\nu_{\tau}+\bar{\nu}_{\mu}$ fluxes. The emergent $\nu_{\tau}$ flux is unity for all energies. We use the density profile given in the Preliminary Reference Earth Model described in, e.g., Ref. [8]. Note the mantle-core transition at $\psi \simeq 33^{\circ}$, corresponding to a radial distance of $3480 \mathrm{~km}$.

the fact that the secondary $\bar{\nu}_{\mu}$ are about $1 / 2$ as energetic as the regenerated $\nu_{\tau}$, and so have a longer interaction length than assumed in the analytic estimate. They are created in the last few interaction lengths of the $\nu_{\tau}$. We find that the $\nu_{\tau}$ and $\bar{\nu}_{\mu}$ are distributed around the transparency energy, as expected. The surviving $\nu_{\mu}$ have the initial energy $E_{0}$, at least until their flux is greatly suppressed. A very small fraction will be moderated down to the transparency energy by repeated NC interactions; this is why the $\nu_{\mu}$ flux in Fig. 2 never completely vanishes.

\section{CONCLUSIONS}

Earth absorbs high-energy $\nu_{e}$ and $\nu_{\mu}$. In fact, highenergy $\nu_{\tau}$ are absorbed too, but the $\nu_{\tau}$ flux is regenerated by prompt tau decays, thus moderating the $\nu_{\tau}$ energies down to near the transparency energy. As a function of the nadir angle, the $\nu_{e}$ and $\nu_{\mu}$ fluxes are exponentially absorbed, while the $\nu_{\tau}$ flux remains unchanged by passage through Earth. Halzen and Saltzberg have proposed that these $\nu_{\tau}$ can be detected by CC interactions after which the tau decays to a muon below the detector, and that they can be separated from $\nu_{\mu} \mathrm{CC}$ interactions by their characteristic nadir-angle dependence [7]. A difficulty with this technique is the $18 \%$ branching fraction for the tau decay into a muon.

We have pointed out a new effect, that the $\nu_{\tau} \rightarrow \tau \rightarrow \nu_{\tau}$ regeneration process creates a secondary $\bar{\nu}_{\mu}$ flux. Though their flux is at most 0.2 of the $\nu_{\tau}$ flux, they are as detectable by the production of muons as the entire $\nu_{\tau}$ flux. Similarly, a secondary $\bar{\nu}_{e}$ flux is created that doubles the detectability of $\nu_{\tau}$ by the production of electrons and their associated showers. Also, the detectability of $\nu_{\tau}$ by neutral-current channels will be about $40 \%$ larger than if the secondary antineutrinos were not produced. For astrophysical antineutrinos, the secondary neutrinos created have the same effect, again doubling the detectability of the $\bar{\nu}_{\tau}$ flux. Taking these secondary fluxes into account in the energy and nadir-angle distributions (e.g., in studies like Refs. [10,14]) will be essential for understanding the spectra and flavor composition of astrophysical neutrinos.

\section{ACKNOWLEDGMENTS}

We thank Francis Halzen, Dan Hooper, David Saltzberg, and Ina Sarcevic for useful discussions. J.F.B. and E.W.K. were supported by NASA under NAG5-10842, and by Fermilab, which is operated by URA under DOE contract No. DE-AC02-76CH03000. E.W.K. thanks the Korea Institute for Advanced Study for their hospitality while this work was completed. P.C. was supported by DOE grant No. 5-90098.
[1] T.K. Gaisser, F. Halzen, and T. Stanev, Phys. Rep. 258, 173 (1995); 271, 355(E) (1995).

[2] J.G. Learned and K. Mannheim, Annu. Rev. Nucl. Part. Sci. 50, 679 (2000).

[3] L. Pasquali, M.H. Reno, and I. Sarcevic, hep-ph/9905389.

[4] Y. Fukuda et al., Phys. Rev. Lett. 81, 1562 (1998); 85, 3999 (2000).

[5] I.F. Albuquerque, L. Hui, and E.W. Kolb, Phys. Rev. D 64, 083504 (2001).

[6] J.H. MacGibbon, U.F. Wichoski, and B.R. Webber, hep-ph/0106337.

[7] F. Halzen and D. Saltzberg, Phys. Rev. Lett. 81, 4305 (1998).

[8] R. Gandhi, C. Quigg, M.H. Reno, and I. Sarcevic, Astropart. Phys. 5, 81 (1996); Phys. Rev. D 58, 093009 (1998).
[9] S. Ritz and D. Seckel, Nucl. Phys. B304, 877 (1988).

[10] S.I. Dutta, M.H. Reno, and I. Sarcevic, Phys. Rev. D 62, 123001 (2000).

[11] T. K. Gaisser, Cosmic Rays and Particle Physics (Cambridge University Press, Cambridge, England, 1992).

[12] J.G. Learned and S. Pakvasa, Astropart. Phys. 3, 267 (1995).

[13] S. Iyer Dutta, M.H. Reno, I. Sarcevic, and D. Seckel, Phys. Rev. D 63, 094020 (2001).

[14] P. Jain, J.P. Ralston, and G.M. Frichter, Astropart. Phys. 12, 193 (1999); F. Becattini and S. Bottai, ibid. 15, 323 (2001); H. Athar, M. Jezabek, and O. Yasuda, Phys. Rev. D 62, 103007 (2000); I.F. Albuquerque, J. Lamoureux, and G.F. Smoot, hep-ph/0109177. 